\title{
La literatura y formas de ver su enseñanza
}

\section{Elizabeth Rojas Samperio*}

Resumen: En este trabajo se presentan un conjunto de reflexiones en torno al papel que juega la literatura en el aprendizaje de una lengua, especialmente en la enseñanza del español como segunda lengua. Se parte de relacionar la literatura como objeto de estudio con su didáctica, en tanto objeto de enseñanza y aprendizaje a partir de cuatro momentos: la época denominada clásica que comprende el curriculum basado en el positivismo y con ello en el modelo enciclopédico; el ingreso del estructuralismo a México, como foco de atención en la enseñanza de lenguas, incluyendo el papel de la literatura como objeto de comentario literario; en un tercer momento, la enseñanza de la literatura como tema de reflexión y de ahí al paso de la forma en que se concibe actualmente la enseñanza de la literatura en el marco de los programas por competencias.

La literatura ha dejado de ser un objeto de estudio para formar parte de una tipología textual en donde el modelo es uno más entre otros. Además, se percibe en el entorno de la competencia lectora. Sin embargo, es tiempo de revalorar su papel como el texto que por sí mismo es privilegiado en tanto en sí mismo ocupa las dos áreas cerebrales, la izquierda para comprender, en tanto texto lineal y la derecha para interpretarlo, como un texto que toca las fibras más profundas del ser humano.

Palabras clave: Literatura, didáctica, comentario literario, comunicación, competencias.

\footnotetext{
* La autora es doctora en Filosofía con la especialidad en Psicología por Atlantic International University. Cuenta con 40 años de experiencia docente en instituciones privadas y públicas. Autora y coautora de diversas publicaciones en las áreas del lenguaje y la comunicación en la Universidad Nacional Autónoma de México y en la Universidad Pedagógica Nacional. Actualmente colaboradora en la autoría de los libros de texto gratuito de Español para la Educación Básica, México, Secretaría Educación Pública y en la elaboración de material digital para la página www.hdt.sep. gob.mx dirigida a maestros y estudiantes de secundaria.
} 
Abstract: In this paper we present a set of reflections on the role of literature in language learning, especially in teaching Spanish as a second language. It starts to relate the literature as a subject of study with his teaching, while teaching and learning object from four periods: the period comprising the so-called classical curriculum based on positivism and encyclopedic by the model, the income structuralism to Mexico as a focus on language teaching, including the role of literature as an object of literary commentary, in a third stage, the teaching of literature as a subject of reflection and hence the passage of the way currently conceived teaching of literature in the context of competency-based programs.

The literature is no longer an object of study to be part of a text typology where the model is one among others. In addition, it is perceived in the environment of literacy skills. However, it is time to reassess its role as the text itself is privileged both in itself occupies two brain areas, the left to understand, as linear text and the right to interpret it as a text that touches the deepest fibers of human beings.

Key words: Literature, teaching, commentary, literary, communication, skills. 


\begin{abstract}
El texto literario no está acabado en sí mismo hasta que el lector lo convierte en un objeto de significado, el cual será necesariamente plural.
\end{abstract}

Roland Barthes ${ }^{1}$

La literatura como objeto de estudio ha pasado por distintas maneras de mirarse y por tanto de presentarse como objeto de estudio y por ende de enseñanza. Sin embargo, las distintas posturas que aquí se describirán coexisten actualmente, en el curriculum, y más especialmente, en la práctica docente cotidiana en casi todos los niveles escolares y superiores.

La literatura es una revelación lingüística que centrada en la función poética de la lengua es una serie de manifestaciones que permiten comprender las funciones de la lengua en su totalidad, penetrando no sólo en los signos y su significado sino en la multiplicidad de sentidos y emergencias. En la literatura se encuentra la historia y la historia del hombre y de la mujer, se comprende al ser, la esencia de la vida y de la muerte. La fuerza indómita de la locura y la serenidad de las emociones humanas en procesos de manifestación y exaltación.

La lengua tiene como función comunicar y representar. Es imagen del pensamiento y señala la cosmovisión de sus hablantes. Es una representación interna de la realidad, construida por medio de la comunicación de los códigos sociales, de actuación social y personal en los planos de relación de los sujetos. Aquí, la literatura representa la socialización de las emociones, de las fantasías y de los deseos más sublimes del ser humano. En la literatura se encuentra la palabra y en la palabra el ser, el significado y el sentido de lo ético y de lo valioso para los hombres y las mujeres que se potencian como lectores de este ente particular y especial. Leer es entonces, un acto fino de comunicación y sentido de los códigos interpretados y las acciones reflejadas en la proyección del lector hacia los acontecimientos o sentimientos del texto literario. Sin embargo, no siempre se ha visto de esta manera el texto literario. Antes bien, se ha visto sobre él, un estudio estatizado.

Así, en la época del ingreso del positivismo al curriculum mexicano, a principios del siglo xx, se concibió a la enseñanza de la literatura como un proceso histórico que debía describir épocas y lugares y con ellas movimientos artísticos y sus manifestaciones en biografía y selección de fragmentos de textos de diferentes autores. El conjunto de situaciones didácticas daban predominio a la descripción de lineamientos sobre la lectura como un compendio de niveles, de pasos, ubicados en un desarrollo histórico social, donde el papel del agru- 
pamiento y memorización de datos cobraba un papel fundamental en el trabajo en la escuela.

Las evaluaciones en este modelo se centraban en la información instalada en la memoria con la posibilidad de reproducción e identificación de características de los movimientos o de las manifestaciones artísticas.

Los programas, entonces, se organizan por épocas históricas y ocasionalmente por lugares o manifestaciones de género. Así encontramos una cantidad de denominaciones curriculares destinadas a señalar estos aspectos: Literatura de los siglos de oro, Literatura mexicana, Cuento latinoamericano. El fin es dar a conocer los procesos históricos reconocidos como tales y ante ellos, las manifestaciones literarias significativas. Pocas veces se observa una teoría lingüística y literaria que sustente estos programas, por lo que, la propia división resulta arbitraria y discrecional. La enseñanza por su parte, se torna expositiva, observando una visión del dominio de "la palabra", del maestro en su poder. (Eguinoa, 2009) La clase se destina más al control disciplinario que a la propia enseñanza, menos aún al aprendizaje. La mayoría de los estudiantes adquieren una postura pasiva de memorización de datos e informaciones pocas veces significativas. De esta manera, sólo una porción mínima de estudiantes se adhiere a la búsqueda por saber más acerca de la literatura, en tanto, la mayoría deserta de ella dirigiendo sus intereses hacia otros ámbitos.

Hacia la década de los setentas, se introduce en México algunas variantes, tanto en los programas de Literatura, como en la propia didáctica. La primera asume que su aprendizaje se debe reflejar en la elaboración de un comentario escrito en el que se aplique una corriente literaria que permita descubrir la estructura básica y particular de cada texto. Se inicia una búsqueda porque el texto sea analizado en sí mismo, a través de los recursos de la lingüística, semióticos principalmente. En el curriculum oculto el ayer no se ha olvidado y los sistemas de clasificación siguen siendo los mismos. Por su parte, la didáctica se ve influenciada por el conductismo y los conceptos que de él se derivan: el aprendizaje representa un cambio de conducta observable; el conocimiento se puede fragmentar y se alcanza por medio de objetivos generales, particulares y específicos; se desarrolla la posibilidad de aprender por medio de la oferta al estudiante para que por medio del ensayo-error, camine por el conocimiento, con errores, pero corrigiendo conforme alcanza niveles taxonómicos superiores.

Se incorporan en la escuela las entonces incipientes tecnologías de la comunicación y la dinámica de grupos desde la perspectiva de la teoría de sistemas. El nivel de especificidad de los objetivos, permitía generar cuadros amplios de literatura comparada en aspectos específicos de los comentarios, cuyo propósito era aplicar un análisis, a veces minucioso de los textos literarios en sí mismos. Si bien, la contextualización de autor y obra se consideraba el primer paso del 
análisis y comentario de textos. Lo cual significa que el pasado aún no se olvida y no se logra trabajar el texto por el texto mismo.

Esta didáctica se ve influida años adelante, por visiones que buscaban destruir los conceptos anteriores. El enfoque situacional comunicativo y los avances de la lingüística aplicada se vierten a los programas de estudios, aunque las prácticas docentes siguen sin cambiar. La lingüística aplicada comprende una serie de disciplinas que apoyan su desarrollo; nace en Estados Unidos hacia 1940 y es, en la Universidad de Michigan donde se le da el nombre años más adelante. Su origen está ligado a la enseñanza de segundas lenguas, elemento que se convirtió en una necesidad ante las situaciones que se estaban viviendo en la Segunda Guerra Mundial. De esta necesidad, que más adelante se torna económica, se inician desarrollos de distintos métodos y enfoques para la enseñanza de lenguas. Se ve afectado tanto el trabajo con $\mathrm{L} 1$ como con $\mathrm{L} 2$, incluso, en espacios considerados específicamente bilingües.

Algunas disciplinas que apoyan el desarrollo de esta postura son la Logopedia como un apoyo a encontrar soluciones para los trastornos de la pronunciación y la oralidad. Desde luego que la pragmática, como rama de la sociolingüística ocupa un papel fundamental; los registros del habla se sitúan en los espacios y contextos para que quien ap3rende una legua le dé un significado más allá de los aspectos que pudiera ofrecer la gramática. De la psicología, se toman los trabajos relacionados con la psicología cognitiva, que se encarga de estudiar los procesos del pensamiento: percepción, elaboración, y procesamiento de información.

Se concibe la enseñanza como un espacio situacional que busca que el alumno se comporte física, moral y lingüísticamente, resolviendo lograr el efecto en su interlocutor y provocar la respuesta deseada. Se parte de reconocer en el estudiante una serie de capacidades que le permiten interactuar con la lengua, objeto de aprendizaje, desde el momento en que ingresa al un aula. Se busca, entonces, que el alumno, a partir de lo que conoce, construya nuevos conocimientos, tanto para expresarlo, como para adquirir los propios conocimientos. No olvidemos que la lengua es un reflejo de la realidad, de ahí que ponerle nombre a una cosa, no sea sólo aprender el nombre, sino aprehender la cosa misma.

Para aprender una lengua y alcanzar en su aprendizaje un empleo estándar de ella, se requiere contar con una serie de estrategias en distintos niveles:

- De comunicación, lo que implica conocer los requisitos del contexto para interpretar y expresar con las palabras y los significados adecuados a la situación.

- Cognitivas. Es decir, circunstancias que permiten aprender.

- Socioafectivas. Relacionadas directamente con la capacidad de involucrarse con los otros, identificando sus necesidades, sin menoscabo de las propias. 
- Metacognitivas. Relacionadas directamente con la comunicativas y socioafectivas; permiten realizar los procesos de autorregulación; en los distintos niveles de uso de la lengua: elección del vocablo respecto de su adecuación, pertinencia, claridad y corrección.

La influencia de los trabajos de Vigostky (1995) y Luria (1993), acerca de la forma en que los procesos de aprendizaje toman y tienen sentido en el mundo que rodea al conjunto de interacciones en las que se participa, adquiriendo un papel fundamental la mediación del que sabe, respecto del que está aprendiendo. Incluso, Reuven Feurestein (2002) crea la Teoría de la Mediación como un elemento básico para explicar los procesos de enseñanza aprendizaje, que establecen las personas cuando de manera intencional buscan formar a otro sujeto.

Se pone en juego, entonces, el debate de los métodos y enfoques para enseñar una lengua, basados tanto en las teorías sobre la lengua, como en las teorías de aprendizaje. Los primeros métodos apoyados en la fonética y la fonología se denominan auditivo-oral y audiolingual. Pronto son superados por el enfoque comunicativo funcional y el enfoque natural.

El enfoque comunicativo funcional, parte de la creación de situaciones comunicativas reales que permiten el desarrollo de las capacidades comunicativas: expresión oral, expresión escrita, competencia auditiva, competencia lectora e interacción social.

En tanto, el método natural parte de la idea de que la lengua se aprende cuando adquiere un significado en el ambiente en que se produce. Terrell, (1977:325-337) afirma que la atención en el proceso de enseñanza está en la importancia del vocabulario y las situaciones de uso, desplazando a la gramática en un segundo término. No se olvide, que años atrás, se habían desarrollado los métodos que proponían el aprendizaje de las lengua a través del trabajo con sus gramáticas. La gramática transformacional adquiere un importante papel, cuando Chomsky (1971) difunde su concepto de gramática universal, como un conjunto de normas básicas que se dan en las estructuras gramaticales de todas las lenguas; por ejemplo, el sentido de género que adquieren las palabras, relacionado directamente, con los conceptos de dominio y descalificación de uno y otro género.

Para crear las situaciones comunicativas, se desarrolla las teorías de los tipos textuales. Entre las dos más difundidas está la Jackobson (1975) basado en las funciones lingüísticas que parten de las intenciones de quien se expresa. Las intenciones se centran en cada uno de los elementos del circuito de la comunicación.

La referencial se ubica en el objeto; la emotiva en los rasgos del emisor, en tanto la apelativa se relaciona con los intereses del receptor. La fática se ubica en el canal, la metalingüística en el código y la poética en el mensaje. Aunque un texto puede incluir varias funciones, generalmente, hay predominio de una de ellas. El foco de atención determina, entonces, el tipo textual (Zacaula, 2000). 
Otros autores han trabajado aspectos relacionados con la tipología textual. Halliday (1982) desde la perspectiva de su gramática sistémica funcional, propone las macrofunciones: ideativa, de relación con el mundo; interpersonal y textual; y las macrofunciones que se determinan por el uso concreto y real. Este uso puede ser extensivo, según las culturas, pero en términos generales se da en el desarrollo de la persona, desde que establece contacto con el mundo. Estas macrofunciones son consecutivas y se dan secuencialmente: instrumental, reguladora, interaccional, personal, heurística, imaginativa, informativa; pragmática, patética (que permite aprender); ideacional, preposicional, interpersonal y finalmente textual.

Los textos, entonces, están determinados por la intención del emisor, respecto de lo que espera del receptor. De ahí, que también se diera lugar a la Teoría de la Recepción donde se destacan la postura de la Escuela de Constanza, y, la versión de Praga de Mukarovsky que determina que la función estética depende del gusto y éste cambia con el tiempo y el conocimiento de las personas.

Entonces, a pesar de los avances en los estudios sobre la lengua, la literatura empieza a perder gran terreno, pierde su carácter de disciplina para convertirse en un tipo textual más, y se deja en su lugar algo difuso denominado "recreación literaria", dando a la literatura un papel meramente decorativo.

Actualmente, se trata de colocar en los programas el desarrollo de competencias como eje central. Este término tiene un carácter polisémico y se presta a varias interpretaciones. La que ha empleado el curriculum mexicano tiene una representación genérica. Se concibe como el conjunto de conocimientos, habilidades, destrezas, aptitudes que se ponen en juego en una situación problemática con el fin de reconocer dicha situación, identificar sus constitutivos y hacerles frente o establecer estrategias de solución en las que se puedan evaluar los riesgos y las posibilidades de éxito.

Hay varios niveles de comprensión de estas competencias:

- Competencias sociales. Colaborar con otras personas en forma constructiva mostrando un comportamiento orientado al grupo y a la comprensión y respeto por el otro.

- Competencias de participación. Saber interactuar, comprendiendo las diferencias individuales y el pensamiento divergente como real.

- Competencias metodológicas. Saber reaccionar encontrando las formas de solución y la transferencia de las experiencias de otros a la resolución de los conflictos.

- Competencias técnicas. Dominio de tareas y contenido en el ámbito en el que se actúa con mayor aceptabilidad. 
El desarrollo de las competencias requiere ser comprobado en la práctica mediante el cumplimiento de desempeños claramente establecidos. Los criterios de desempeño, entendidos como los aprendizajes esperados en términos de productos de aprendizaje (evidencias), establecidas las condiciones para determinar la calidad del desempeño (criterios y valores) son la base para evaluar que se alcanzó determinada competencia. Por eso, los criterios de evaluación están estrechamente ligados a la práctica didáctica.

El modelo por competencias educativas establece tres niveles, cuyos rangos de generalidad van de lo general a lo particular.

1. Competencias básicas. Son las capacidades intelectuales y manuales mínimas para acceder a un conocimiento. En ellas se encuentran competencias cognitivas, técnicas y metodológicas de acción.

2. Competencias genéricas. Son la base de un conocimiento y se refieren a situaciones concretas de la disciplina que por si mismas, demandan de respuestas complejas.

3. Competencias específicas. Son la base del ejercicio profesional y están vinculadas a situaciones específicas de actuación.

La literatura pasa a ser parte de la competencia lectora y es sólo una pequeña parte de las competencias que se necesitan en una economía basada en la sociedad del conocimiento; a saber:

- Navegación en el conocimiento: a dónde ir y cómo procesar la información.

- Buenas competencias comunicativas / leer, escribir, hablar, escuchar).

- Capacidad de aprender de forma independiente.

- Competencias sociales: ética, actitudes positivas, responsabilidad.

- Trabajo en equipo.

- Capacidad de adaptarse a circunstancias cambiantes.

- Destrezas de razonamiento: resolución de problemas, capacidad crítica, lógica, numérica.

- Iniciativa personal y voluntad de intentar cosas nuevas (ser emprendedor). (Bautista, 2008)

La literatura se coloca sólo como una franja muy pequeña de la sociedad en las competencias específicas. En la escuela, es un lujo, algún día afirmaría Bertrand Russell (1974) “¿Deben estudiar los jóvenes preferentemente clásicos o ciencia? Lo que se dice más corrientemente es lo que los clásicos son ornamentales y la ciencia es útil. ¿Se debe enseñar a los niños a hablar correctamente y a tener bue- 
nas maneras, o esto no es otra cosa que restos de aristocratismo? Discusión entre quienes quieren conservar los valores de la aristocracia y los que se adhieren al nuevo pensamiento democrático.

Difícil postura, cuando se quiere ver como una persona democrática y al tiempo, defender el papel del pensamiento y de su expresión en la literatura. Como consecuencia de ello, es que el aprendizaje de la literatura se queda en como una parte de la competencia lectora.

El estudio de la literatura reducida a un modelo de tipo textual en el que compite espacios de interlocución con otros tipos textuales: científicos, de interacción social, instructivos, legales deja poco espacio para la reflexión de las bondades que tiene la literatura en sí misma. El predominio se dirige a identificar los elementos estructurales del texto a fin de servir de modelo para la construcción de textos propios, aunque no se pueda diferenciar cuando el texto sólo puede ser leído, pero no copiado. Lo importante es adquirir la competencia lectora, de distintos textos, entre ellos el literario, asumiendo la capacidad para leer como un trabajo con el pensamiento; interacción de pensamientos entre el autor y el lector. Incluso, hasta poder valorar el texto mismo, para su recomendación o para "comentarlo" desde el gusto mismo del lector.

La Organización para la Cooperación y el Desarrollo Económicos (OCDE) define como competencia lectora la capacidad para comprender, emplear la información y reflexionar en torno a los textos escritos, con el fin de lograr sus metas individuales, desarrollar sus conocimientos y potencial personal, y participar en la sociedad.

La lectura, desde la perspectiva del proyecto PISA, supera el concepto tradicional de descodificación y comprensión literal y busca alcanzar la comprensión y con ella la interpretación de los mensajes textuales. Considerando:

\begin{tabular}{|c|l|}
\hline \multirow{3}{*}{ Procesos } & $\begin{array}{c}\text { 1. Recuperación de información. } \\
\text { Localizar información relevante, da- } \\
\text { tos aislados o vinculados con otros } \\
\text { elementos del texto. } \\
\text { 2. Interpretación de textos. Encon- } \\
\text { trar el significado y llegar a alguna } \\
\text { conclusión a partir del texto escrito. } \\
\text { 3. Reflexión y evaluación de textos. } \\
\text { Relacionar la forma escrita en cuanto } \\
\text { forma y contenido, con sus conocimien- } \\
\text { tos, ideas y experiencias anteriores. }\end{array}$ \\
\hline
\end{tabular}




\begin{tabular}{|c|c|}
\hline \multirow{5}{*}{ Formato textual } & $\begin{array}{c}\text { 1. Los textos continuos están orga- } \\
\text { nizados en oraciones y párrafos. Se } \\
\text { clasifican por su tipo y por la inten- } \\
\text { ción del autor. } \\
\text { 2. Los textos discontinuos presentan } \\
\text { la información en formas muy variadas, } \\
\text { por los que se clasificó más por su es- } \\
\text { tructura que por la intención del autor. } \\
\text { 1. Una carta, una novela o una bio- } \\
\text { grafía están destinadas a un uso priva- } \\
\text { do (personal). } \\
\text { 2. Los anuncios o documentos ofi- } \\
\text { ciales tienen un uso público. } \\
\text { 3. Un manual o un informe son para } \\
\text { uso laboral (profesional). } \\
\text { 4. Un libro de texto u hoja de ejer- } \\
\text { cicios están destinados para su uso } \\
\text { educativo. }\end{array}$ \\
\hline
\end{tabular}

Como se puede apreciar la literatura se concibe como un texto de carácter personal que se presta a tantas interpretaciones como lecturas tenga. Incluso, si las lecturas se realizan por la misma persona. Aunque se recuperan ejemplos de los distintos géneros literarios, ya nos son importantes ni los autores, ni los momentos de producción, sino específicamente el tiempo de recepción que parte del momento en que el texto es leído. Es decir, lo importante es el contexto del lector y no del texto.

Se busca que los estudiantes adquieran una competencia lectora independientemente del texto que estén leyendo. 


\begin{tabular}{|c|c|c|c|}
\hline \multicolumn{4}{|c|}{ Los niveles de actuación frente a la lectura, dictados por la oCDE } \\
\hline Nivel & $\begin{array}{l}\text { Recuperacion de } \\
\text { la información }\end{array}$ & $\begin{array}{l}\text { Interpretacion de } \\
\text { textos }\end{array}$ & $\begin{array}{l}\text { evaluación de } \\
\text { textos }\end{array}$ \\
\hline 5 & $\begin{array}{l}\text { Localizar y posible- } \\
\text { mente ordenar o com- } \\
\text { binar varios fragmen- } \\
\text { tos de información } \\
\text { que no resultan evi- } \\
\text { dentes en absoluto, } \\
\text { algunos de los cuales } \\
\text { pueden encontrarse } \\
\text { fuera del corpus prin- } \\
\text { cipal del texto. Inferir } \\
\text { que información del } \\
\text { texto es relevante } \\
\text { para la tarea. Mane- } \\
\text { jar información muy } \\
\text { verosímil y/o abun- } \\
\text { dante información en } \\
\text { conflicto. }\end{array}$ & $\begin{array}{l}\text { Interpretar el } \\
\text { significado de un } \\
\text { lenguaje lleno de } \\
\text { matices o demostrar } \\
\text { una comprensión } \\
\text { completa del texto. }\end{array}$ & $\begin{array}{l}\text { Valorar de manera } \\
\text { crítica o formular } \\
\text { hipótesis haciendo } \\
\text { uso de conocimien- } \\
\text { tos especializados. } \\
\text { Manejar conceptos } \\
\text { contrarios a las expec- } \\
\text { tativas y hacer uso } \\
\text { de una comprensión } \\
\text { profunda de textos } \\
\text { largos y complicados. }\end{array}$ \\
\hline 4 & $\begin{array}{l}\text { Localizar y posible- } \\
\text { mente ordenar o } \\
\text { combinar varios } \\
\text { fragmentos de infor- } \\
\text { mación que resulten } \\
\text { evidentes, que es } \\
\text { posible que tengan } \\
\text { que ajustarse a varios } \\
\text { criterios, en un } \\
\text { texto cuyo contexto } \\
\text { o forma resulten } \\
\text { habituales. Inferir } \\
\text { que información del } \\
\text { texto es relevante } \\
\text { para la tarea. }\end{array}$ & $\begin{array}{l}\text { Utilizar un nivel } \\
\text { elevado de inferencia } \\
\text { basada en el texto } \\
\text { para comprender y } \\
\text { aplicar categorías en } \\
\text { un contexto poco } \\
\text { habitual e interpretar } \\
\text { el significado de una } \\
\text { sección del texto } \\
\text { teniendo el cuenta el } \\
\text { texto en su totalidad. } \\
\text { Manejar ambigüe- } \\
\text { dades, ideas contrarias } \\
\text { a las expectativas e } \\
\text { ideas expresadas en } \\
\text { forma negativa. }\end{array}$ & $\begin{array}{l}\text { Utilizar conocimientos } \\
\text { públicos o formales } \\
\text { para formular hipótesis } \\
\text { o analizar de manera } \\
\text { crítica un texto. } \\
\text { Mostrar una compren- } \\
\text { sión precisa de textos } \\
\text { largos y complicados. }\end{array}$ \\
\hline
\end{tabular}




\begin{tabular}{|c|c|c|c|}
\hline \multicolumn{4}{|c|}{$\begin{array}{l}\text { Los niveles de actuación frente a la lectura, dictados por la OCDE } \\
\text { (continuación) }\end{array}$} \\
\hline Nivel & $\begin{array}{l}\text { Recuperación de } \\
\text { la información }\end{array}$ & $\begin{array}{l}\text { Interpretación de } \\
\quad \text { textos }\end{array}$ & $\begin{array}{l}\text { Reflexión y } \\
\text { evaluación de } \\
\text { textos }\end{array}$ \\
\hline 3 & $\begin{array}{l}\text { Localizar y en algu- } \\
\text { nos casos reconocer } \\
\text { la relación entre los } \\
\text { distintos fragmen- } \\
\text { tos de información } \\
\text { que es posible } \\
\text { que tengan que } \\
\text { ajustarse a varios } \\
\text { criterios. Manejar } \\
\text { información impor- } \\
\text { tante en conflicto. }\end{array}$ & $\begin{array}{l}\text { Integrar distintas } \\
\text { partes de un texto } \\
\text { para identificar } \\
\text { una idea principal, } \\
\text { comprender una } \\
\text { relación o interpre- } \\
\text { tar el significado de } \\
\text { una palabra o frase. } \\
\text { Comparar, } \\
\text { contrastar o catego- } \\
\text { rizar teniendo en } \\
\text { cuenta muchos crite- } \\
\text { rios. Manejar infor- } \\
\text { mación en conflicto. }\end{array}$ & $\begin{array}{l}\text { Realizar conexiones } \\
\text { o comparaciones, dar } \\
\text { explicaciones o valorar } \\
\text { una característica del } \\
\text { texto. Demostrar un } \\
\text { conocimiento detalla- } \\
\text { do del texto en relación } \\
\text { con el conocimiento } \\
\text { habitual y cotidiano } \\
\text { o hacer uso de cono- } \\
\text { cimientos menos } \\
\text { habituales. }\end{array}$ \\
\hline 2 & $\begin{array}{l}\text { Localizar uno o } \\
\text { más fragmentos de } \\
\text { información que es } \\
\text { posible que ten- } \\
\text { gan que ajustarse } \\
\text { a varios criterios. } \\
\text { Manejar información } \\
\text { en conflicto. }\end{array}$ & $\begin{array}{l}\text { Identificar la idea } \\
\text { principal del texto, } \\
\text { comprender rela- } \\
\text { ciones, construir o } \\
\text { aplicar categorías } \\
\text { simples u obtener el } \\
\text { significado con una } \\
\text { parte limitada del } \\
\text { texto cuando la in- } \\
\text { formación buscada } \\
\text { no es evidente y se } \\
\text { requieren inferen- } \\
\text { cias sencillas. }\end{array}$ & $\begin{array}{l}\text { Hacer una compara- } \\
\text { ción o conectar el } \\
\text { texto y el conocimiento } \\
\text { externo, o explicar una } \\
\text { característica del texto } \\
\text { haciendo uso de ex- } \\
\text { periencias y actitudes } \\
\text { personales. }\end{array}$ \\
\hline
\end{tabular}




\begin{tabular}{|c|l|l|l|}
\hline \multicolumn{3}{|c|}{ Los niveles de actuación frente a la lectura, dictados por la OCDE } \\
(continuación)
\end{tabular}

Se espera que el lector alcance una competencia suficiente hacia los 15 años, lo que nos permite identificar qué debe saber hacer un adulto universitario y especialmente un alumno que busque el conocimiento de las letras como objeto de estudio.

La didáctica se torna participativa, el docente deja de ser eje de atención para girar la atención en torno al estudiante, erigiéndose el docente como el líder experto que resuelve las dudas de su alumno y dirige las actuaciones frente al objeto de conocimiento. Sin embargo, la práctica sigue siendo decimonónica, el docente es el eje, la historia de la literatura sigue siendo el objeto de estudio y su aprendizaje se torna enciclopédico.

¿Cuándo podremos dar un salto cuantitativo hacia el terreno internacional y no quedarnos en los siglos anteriores, defendiendo la tradición por encima de la innovación? Es tiempo de reflexionar y buscar nuevas opciones del estudio de la literatura.

Si como expresé al principio de este escrito, la literatura es algo más que palabras. ¿Cuándo el estudiante estará dispuesto a trabajar con el texto hasta los niveles de excelencia? ¿Cuándo el maestro se atreverá a dejar a su alumno experimentar, bucear, penetrar en el texto aunque llegue a conclusiones diferentes? ¿Cuándo el curriculum abrirá sus puertas al siglo xxı?

No creo que saber literatura sea un lujo. Menos aún si la vemos como una herramienta para conocer y comprender el espacio y el tiempo en que se produce la obra, el reflejo que hace de la realidad misma y la forma en que a través de ella se permite conocer el sentir y pensar de los pueblos. 
Cuando se emplea su estudio en el aprendizaje de L2, cobra, incluso, una importancia mayor. Si como hemos venido afirmando, las lenguas se aprenden en la interacción de las personas y el conocimiento se adquiere al dialogar con los otros, ¿qué mejor que dialogar con los pensadores que recopilan toda su sabiduría en el texto que presentan para disfrutar y reflexionar sobre los hombres y sus cosas, sus intereses, sus deseos, sus problemas, su manera de ver el mundo.

Queden estas últimas preguntas como reflexiones finales en torno a la enseñanza de la literatura.

La postura personal es que ninguna persona que se precie de conocer el mundo, podrá afirmarse como tal si no tiene contacto con la literatura. La literatura que demanda su espacio propio para entrar a las puertas del alma y dormirse en la vela del desvelo y en el sueño de los deseos más profundos y brillantes.

BiBLIOGRAFÍA

Conference Board of Canada. (1991) citado por Bautista G. et al. (2008). Didáctica universitaria en entornos virtuales de Enseñanza Aprendizaje. Madrid: Narcea.

Cortés Moreno, M. (2000). Guía para el profesor de idiomas. Barcelona: Ocatedro. Сномsку, N. (1971). Fundamentos de la gramática transformacional. México: Siglo xxı. EguInOA, A. E. (2009). Didáctica de la literatura: proceso comunicativo. Recuperado 8 de marzo de http:// www.uv.mx/cpeu/colección/

FeuresteIN, R. (2002) El desarrollo potencial del aprendizaje. Entrevista.

Gracida JuÁrez, Y. y Ruiz Ocampo A. (2004). Competencia comunicativa y diversidad textual. México: Edere.

JACKOBSON, R. (2000) citado por Zacaula, F. et al. Lectura y redacción de textos. México: Santillana.

http://cvc.cervantes.es (Recuperado el 15 de mayo de 2011)

http://es.escribid.com (Recuperado el 20 de mayo de 2011)

http://revistas.ucm.es (Recuperado el 12 de mayo de 2011)

M.A.K. Halliday. (1982). El lenguaje como semiótica social. México: Fondo de Cultura Económica.

MARíN, M. (2000). Lingüística aplicada. Madrid: Red síntesis.

Nunan, D. (1998). El diseño de tareas para la clase comunicativa. México: Cambridge University Press.

NuRIA, S. y M. R. (1993). La formación de la función semiótica Psicología evolutiva: infancia y preadolescencia. Barcelona: CEAC. 
Mendoza Fillola, A. (2004). La educación literaria. Bases para la formación de la competencia lecto-literaria. México: Aljibe.

Muñoz, C. E. (2000). Segundas lenguas. Adquisición en el aula. Barcelona: Ariel Lingüística.

PISA en el aula. (2008). México: INEE, Textos de divulgación.

RusselL, B. (1974). Ensayos sobre educación. Madrid: Espasa Calpe. (Col. Austral, 1387).

Santos Gallardo, I. (1999). Enseñanza aprendizaje del español como lengua extranjera. Madrid: Arco/libros.

TERRELL, T. D. (1977). Anatural approach to second lenguaje acquisition and learning. Modem lenguaje Jorurnal, (6) 325-337.

Teoría de la mediación. (2002). citado por Noguez El desarrollo potencial del aprendizaje. Entrevista a Reuven Feurestein. Revista electrónica de investigación educativa, 4, (2). Recuperado el 8 de marzo de 2011 de http://redie.uasb.mx/ vol4no2/contenido noguez.html 